Vladimír Mazocha, Ondřej Mikulaa, , Josef Bryja, Hana Konvičková, Isa-Rita Russo, Erik Verheyen and Radim Šumbera

\title{
Phylogeography of a widespread sub-Saharan murid rodent Aethomys chrysophilus: the role of geographic barriers and paleoclimate in the Zambezian bioregion
}

https://doi.org/10.1515/mammalia-2017-0001

Received January 7, 2017; accepted June 28, 2017

Abstract: Murid rodents of the genus Aethomys are one of the most common rodents in drier habitats in subSaharan Africa. Among them, the red veld rat Aethomys chrysophilus is the most widespread species with the core distribution located in the Zambezian bioregion. In this study, we describe phylogeographic structure of the species and estimate its age from a time-calibrated phylogeny of the genus. Seven parapatric clades were identified in the mitochondrial cytochrome $b$ phylogeny, where some of the distributions of these clades have been separated by previously described biogeographical divides (Zambezi-Kafue river system, Rukwa Rift and the Eastern Arc Mountains). One internal clade corresponded to populations previously described as a distinct species, Aethomys ineptus. The whole $A$. chrysophilus complex was estimated to be $1.3(0.5-2.4)$ Mya old, with $A$. ineptus originating 0.7 (0.1-1.4) Mya before present. The internal position of

aladimír Mazoch and Ondřej Mikula: These authors contributed equally to this article.

*Corresponding author: Ondřej Mikula, Institute of Vertebrate Biology, Czech Academy of Sciences, Květná 8, Brno 60365, Czech Republic; and Institute of Animal Physiology and Genetics, Czech Academy of Sciences, Brno, Czech Republic, e-mail: onmikula@gmail.com

Vladimír Mazoch: Department of Zoology, Faculty of Science, University of South Bohemia, České Budějovice, Czech Republic; and Institute of Vertebrate Biology, Czech Academy of Sciences, Květná 8, Brno 60365, Czech Republic

Josef Bryja: Institute of Vertebrate Biology, Czech Academy of Sciences, Květná 8, Brno 60365, Czech Republic; and Department of Botany and Zoology, Faculty of Science, Masaryk University, Brno, Czech Republic

Hana Konvičková: Institute of Vertebrate Biology, Czech Academy of Sciences, Květná 8, Brno 60365, Czech Republic

Isa-Rita Russo: Cardiff School of Biosciences, Cardiff University, Sir Martin Evans Building, Museum Avenue, Cardiff, CF10 3AX, UK Erik Verheyen: Royal Belgian Institute for Natural Sciences, Operational Direction Taxonomy and Phylogeny, 1000, Brussels, Belgium; and Evolutionary Ecology Group, Biology Department, University of Antwerp, 2020, Antwerp, Belgium

Radim Šumbera: Department of Zoology, Faculty of Science, University of South Bohemia, České Budějovice, Czech Republic
A. ineptus was also recovered in phylogenetic reconstruction based on two nuclear genes and thus it is not a consequence of mitochondrial introgression. In addition, we analyzed skull form variation across the species' distributional range and found no significant difference between $A$. ineptus and the rest of $A$. chrysophilus complex.

Keywords: Aethomys chrysophilus; Aethomys ineptus; phylogeography; Plio-Pleistocene climate changes; Zambezian bioregion.

\section{Introduction}

A recent boom in studies combining molecular genetics and geographical data provides a tool for better understanding phylogenetic diversity and historical processes responsible for the current distribution of species, especially in neglected areas across the globe. For example, in sub-Saharan Africa descriptions of genetic diversity helped to identify historical refugia and migration barriers in larger mammals, e.g. ungulates (Lorenzen et al. 2012), baboons (Zinner et al. 2013) and green monkeys (Haus et al. 2013). Despite substantial progress, we are still far from fully comprehending all factors that have affected the current distribution of biological diversity on the continent. The above-mentioned studies showed low genetic differentiation in large mammals possibly as a result of high historical and/or ongoing gene flow linked with species characteristics such as the ability to survive in a wide range of habitats and the potential for long distance dispersal. In contrast, small mammals represent suitable candidates for the reconstruction of the biotic history on a finer scale due to short generation times, limited dispersal ability and strong associations with particular habitats. For instance, periodic fragmentation of African forests during the Plio-Pleistocene caused by palaeoclimatic changes resulted in remarkable genetic differentiation of forest dwelling small non-flying mammals (e.g. Nicolas et al. 2011, Demos et al. 2014, Bohoussou et al. 2015).

On the other hand, factors affecting the evolution of species living in drier and more open habitats remain less known. Phylogeographic studies suggested the 
importance of rivers and climatic fluctuations in the divergence of genealogical lineages of rodents inhabiting the belt of Sudanian savanna (e.g. Bryja et al. 2010, Dobigny et al. 2013), while different vegetation types could be responsible for the current intraspecific genetic variability in southern Africa (Russo et al. 2010). Recent studies from the eastern African savanna-woodland mosaic illustrated the processes involved in the forming of the fauna of open habitats and the effects of natural barriers (rivers and mountains) in combination with habitat specificity (Nicolas et al. 2008, Colangelo et al. 2013, McDonough et al. 2015, Mikula et al. 2016, Aghová et al. 2017).

In this study, we analyzed the genetic structure of a murid rodent, the red veld rat Aethomys chrysophilus (de Winton 1896). The distribution of this species is tightly linked to the Miombo woodland and the adjacent drier habitats. This species can therefore serve as a suitable model for assessing the role of historical factors that shaped the evolution of African seasonal savanna-woodlands. Among the nine currently recognized Aethomys species, the red veld rat has by far the largest distribution range. This medium-sized rodent with reddish-brown pelage mixed with dark hairs and a long sparsely haired tail occurs from southern-most Kenya through Tanzania, Zambia and Zimbabwe to the KwaZulu-Natal province of South Africa, and from Namibia and southern Angola to southern Mozambique. Across its distribution range it is found in various savanna and woodland habitats, but absent from very arid regions and forests (Linzey and Chimimba 2008).

Gordon and Rautenbach (1980) found two cytotypes ( $2 \mathrm{n}=44$ and 50) in Aethomys chrysophilus and Chimimba (1998) designated $2 n=44$ populations as a separate species Aethomys ineptus (Thomas and Wroughton 1908), given their distinct distribution in southern Africa and very specific sperm morphology (Visser and Robinson 1986, 1987). A more recent study based on mitochondrial DNA suggests that $A$. ineptus is monophyletic, but nested within A. chrysophilus from southern and eastern Africa, so the latter species is left paraphyletic (Russo et al. 2006). In addition, Russo et al. (2006) suggested further in-depth analysis to study genetic differentiation within A. chrysophilus with samples representing the entire distributional range of the species. This study also stressed the need for a taxonomic revision within the species to identify any additional cryptic taxa that may be contained within the currently described $A$. chrysophilus. Here we refer to both species as members of the A. chrysophilus complex.

Using a phylogeographic approach combined with species distribution modeling and morphometric analysis, we aimed to (1) to assess the mitochondrial diversity within the Aethomys chrysophilus complex using the cytochrome $b(C Y T B)$ gene; (2) to identify geomorphological factors and/or climatic processes responsible for the current distribution of mitochondrial lineages; (3) to date divergences of the main mitochondrial lineages and confirm internal position of Aethomys ineptus within the A. chrysophilus complex by analysis of nuclear markers; (4) to test for skull form differences between phylogeographic lineages.

\section{Materials and methods}

\section{Sampling}

We obtained and analyzed genetic information from 222 individuals documented in the Supplemental File 1 available via figshare (DOI: 10.6084/m9.figshare.4516745). Tissue samples (169) were collected by the authors or recovered from museum specimens, namely from collections of Centre de Biologie pour la Gestion des Populations (Montpellier, France), Royal Belgian Institute of Natural Sciences (Brussels, Belgium), Magyar Természettudományi Múzeum (Budapest, Hungary), Muséum National d'Histoire Naturelle (Paris, France), Naturmuseum Senckenberg (Frankfurt, Germany), Smithsonian Institution - National Museum of Natural History (Washington, DC, USA) and Texas Tech University (Lubbock, TX, USA). This material was supplemented by sequences from 23 georeferenced individuals downloaded from the African Rodentia database (http://projects.biodiversity.be/africanrodentia) and 30 individuals from GenBank (http://www.ncbi.nlm. nih.gov/genbank). In total, genetic data were collected from 108 georeferenced localities in 10 countries almost spanning the complete distribution of the Aethomys chrysophilus complex (see Supplemental File 1, Figure 1).

\section{Genotyping}

DNA from fresh (ethanol- or DMSO-preserved) tissues was extracted using the DNeasy tissue kit (Qiagen, Hilden, Germany) following the manufacturer's instructions. For the basic genetic characterization of all individuals we amplified the complete mitochondrial CYTB gene by polymerase chain reaction (PCR) using primers detailed in Table 1. For the purpose of divergence dating and confirmation of the observed mitochondrial diversity, we also sequenced the nuclear interphotoreceptor retinoid binding protein (IRBP) and the recombination activation protein (RAG1) of selected specimens (see more details in Table 1). 


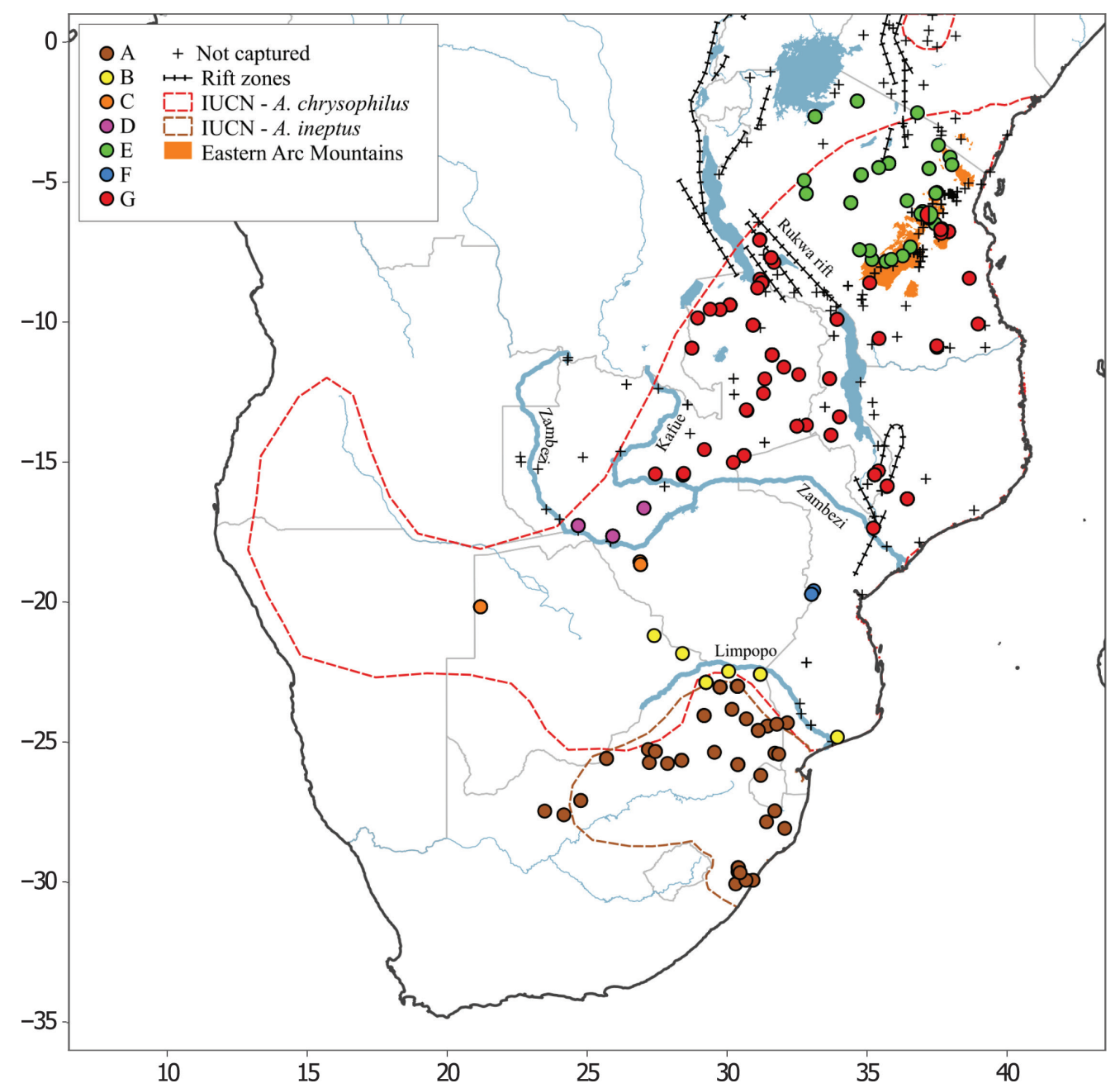

Figure 1: Geographic distribution of seven clades of the $A$. chrysophilus complex.

Trapping sites of particular clades are indicated by color that correspond to Figure 2. Black crosses represent sites where trapping was conducted but no individuals of $A$. chrysophilus complex were captured. Brown (A. ineptus) and red (A. chrysophilus) dotted lines demarcate distribution range according to IUCN. Blue color comprises lakes and main rivers. Black lines showing position of main rift faults.

Table 1: Amplified genetic markers.

\begin{tabular}{llll}
\hline Locus & Primers $\left(\mathbf{5}^{\prime} \rightarrow \mathbf{3}^{\prime}\right)$ & Ta & Reference \\
\hline CYTB & L14723: ACC AAT GAC ATG AAA AAT CAT CGT T & $52^{\circ} \mathrm{C} / 60 \mathrm{~s}$ & Irwin et al. 1991 \\
& H15915: TCT CCA TTT CTG GTT TAC AAG AC & & Stanhope et al. 1992 \\
IRBP & IRBP-217: ATG GCC AAG GTC CTC TTG GAT AAC TAC TGC TT & $55^{\circ} \mathrm{C} / 30 \mathrm{~s}$ & Teeling et al. 2000 \\
RAG1 & IRBP-1531: CGC AGG TCC ATG ATG AGG TGC TCC GTG TCC TG & $\begin{array}{l}60-57^{\circ} \mathrm{C} \\
\text { (touch down PCR) }\end{array}$ & \\
\hline
\end{tabular}

The purified PCR products were sequenced in a commercial laboratory. Genetic data obtained from fresh material were complemented by additional sequences from 10 museum samples (mostly dry skins; see Supplemental File 1), where partial CYTB sequences were generated by pyrosequencing on GS Junior (Roche, Basel, Switzerland) by using the mini-barcode protocol (Galan et al. 2012). The main advantage of this approach is that it allows separating individual sequences in samples contaminated by distantly related organisms (often the case of museum samples), which is not possible through the traditional Sanger sequencing method (for more details see Bryja 
et al. 2014). Obtained sequences were edited and aligned using Geneious version 9.1.5 (Biomatters, Auckland, New Zealand, available from http://www.geneious.com). Unpublished sequences used in our phylogenetic analyses were submitted to GenBank (accession numbers KY965315-KY965392, KU723654, KU723655, KU723662, KU723668, KU723672, KU747156, KU747157).

\section{Phylogenetic reconstructions and historical demography}

The CYTB phylogeny was inferred from 86unique haplotypes using a Bayesian inference and a maximum likelihood (ML) approach the best-fit model of evolution $(\mathrm{GTR}+\mathrm{G})$ was selected using jModelTest 2 (Darriba et al. 2012). The Bayesian analysis in MrBayes 3.2.6 (Ronquist et al. 2012) consisted of two independent Markov chain Monte Carlo (MCMC) runs whose mixing and convergence was checked in Tracer v1.6 (Rambaut and Drummond 2013) with default priors on all parameters. A $10 \%$ burn-in was sufficient to ensure that trees were only sampled after MCMC reached its equilibrium distribution. The ML phylogeny was estimated by RA $\times$ ML 8.6.2 (Stamatakis 2014) using rapid bootstraping (1000 replicates) to evaluate support for internal nodes (Stamatakis et al. 2008). The same three outgroups (Aethomys hindei, Aethomys kaiseri, Aethomys nyikae) were used in both analyses. All phylogenetic and divergence dating computations were performed on the CIPRES cluster (Miller et al. 2010). An alternative view of CYTB variation was provided by the neighbor-net haplotype network method in Splits Tree 4.0 (Huson and Bryant 2006).

Eighty-six haplotypes included in the CYTB phylogeny represented 129 individuals. Remaining shorter sequences from 82 individuals were placed post hoc into the majority consensus Bayesian tree by the evolutionary placement algorithm (EPA; Berger et al. 2011). The EPA accepts the tree topology and sequence alignment as inputs, performs ML estimations of GTR $+\mathrm{G}$ parameters and branch lengths on the fixed topology. Query sequences were then taken one by one to estimate their ML placements in the phylogeny.

We calculated Tajima's D (Tajima 1989) and Fu's $F_{s}(F u$ 1997) statistics for the two clades with more than 10 haplotypes to test whether the observed CYTB variation conformed to expectation for a neutrally evolving locus in mutation-drift equilibrium. In addition, predictions of a sudden expansion model were assessed using mismatch distributions and Harpending raggedness index (Rogers and Harpending 1992). Historical demography statistics were calculated using DnaSP 5.10.1 (Librado and Rozas
2009) and mismatch distributions were visualized using the MMD function as implemented in the $\mathrm{R}$ package “pegas” (Paradis 2010).

\section{Dating of divergences}

The oldest fossil that has been reliably identified as belonging to the genus Aethomys has been found with other Arvicanthini (Arvicanthis, Lemniscomys) in Kenya, Lemudong'o (Manthi 2007), estimated at 6.08-6.12 million years ago (Mya) (Deino and Ambrose 2007). The only other Arvicanthini fossil has been reported by Mein et al. (2004) from Harasib in Namibia. However, the lower molars were very untypical of Aethomys with some uncertainty around the date of this fossil. The faunal composition was similar to Ethiopian Chorora which may be as old as 10-11 Mya (Geraads et al. 2002) or 8.5 Mya (Suwa et al. 2015). Thus, we chose to estimate minimum divergence times by setting a monotonically decreasing calibration for the root of the Aethomys phylogeny. We used an exponential distribution with a mean of 1.504 and an offset of six which gives the highest probability to the minimum age of 6 Mya and only $5 \%$ of the probability density to age older than 10.5 Mya. However, the actual priors of node ages were different due to the inferred fossil calibration density with the birth-death tree shape prior (Heled and Drummond 2012) and we therefore explored this by MCMC sampling. The 95\% probability density was between 6.0 and 9.4 Mya for the root and 0.0 and 5.2 Mya for the most recent common ancestor (MRCA) of Aethomys chrysophilus (or any other monophyletic group of three sequences).

The dating was performed in BEAST 2.3.2 (Bouckaert et al. 2014) using nuclear gene (IRBP, RAG1) sequences of nine Aethomys specimens (see Supplemental File 1), each representing one of the major lineages (species or intraspecific phylogroups) within the genus. More specifically, we included three lineages of the Aethomys chrysophilus complex including Aethomys ineptus to test for its internal placement based on nuclear genes only. Following preliminary analyses in jModelTest 2 we used the K80 model of nucleotide substitution (Kimura 1980). We assumed a relaxed molecular clock with uncorrelated lognormal distribution of evolutionary rates (Drummond et al. 2006) and the same birth-death tree for all genes. Two separate runs were conducted and combined after checking for mixing and convergence with a 10\% burnin. A maximum clade credibility tree was determined using the TreeAnnotator tool of BEAST. Tree figures were produced using R software (R Core Team 2016) using the package “ape” (Paradis et al. 2004). 


\section{Species distribution modeling}

For the species distribution modeling, we merged all georeferenced records of the Aethomys chrysophilus complex from our own and published data sets (Gordon and Rautenbach 1980, Gordon and Watson 1986, Visser and Robinson 1986, 1987, Baker et al. 1988, Breed et al. 1988, Ducroz et al. 2001, Fadda et al. 2001, Castiglia et al. 2003, Linzey et al. 2003, Russo et al. 2006, Nicolas et al. 2011, Phukuntsi et al. 2016, this study) in which species identity was confirmed by mtDNA, karyotypes and sperm morphology (see Supplemental File 1). After discarding samples from identical localities and rounding latitudes and longitudes to $0.5^{\circ}$ we obtained 126 unique records.

The model was built using the MaxEnt algorithm (Phillips et al. 2006). The background was represented by a regular $0.5^{\circ}$ grid of 2986 points covering all known distribution of Aethomys chrysophilus (our data, Galster et al. 2007, IUCN Red List v. 2015-4) and adjacent areas with similar habitats (Olson et al. 2001). As predictors, we used 19 bioclimatic variables obtained from the WorldClim database (Hijmans et al. 2005). The MaxEnt predictions were expressed as relative occurrence rates (RORs; also called the raw output) divided by uniform prior expectation at each background point $\left(\frac{1}{2986}\right)$. RORs were predicted for all background sites as well as for the corresponding sites in layers containing climate reconstructions for the last glacial maximum (LGM; 21,000 years BP, Braconnot et al. 2007) and the last inter-glacial (LIG; $\sim 120,000-140,000$ years BP, Otto-Bliesner et al. 2006). To evaluate the importance of each predictor we randomized them spatially and calculated the Spearman correlation $\left(r_{s}\right)$ of each predictor before and after randomization. The importance was then quantified as $1-r_{s}$.

Model selection using the corrected Akaike information criterion (AICc; Warren and Seifert 2011) was employed to choose predictor transformations (so called features) and values of LASSO regularization coefficient which causes some predictors to have zero regression coefficients and thus effectively removes them from the model (see Merow et al. 2013 for full explanation). Both models in the AICc-based confidence set included linear and quadratic features and thus we used them in combination with a weighted mean of the regularization coefficient $(=1.31)$. We did not attempt to reduce the set of bioclimatic variables, but we relied on the LASSO regularization to select their most appropriate subset.

Species distribution modeling was used as implemented in MaxEnt v.3.3.3.k (Phillips et al. 2006) and interfaced to the $\mathrm{R}$ computing environment ( $\mathrm{R}$ Core Team
2016) by using packages "dismo" (Hijmans et al. 2016) and "ENMeval" (Muscarella et al. 2014). The R script is available as the Supplemental File 2 (DOI: 10.6084/ m9.figshare.4960406). The results were visualized using the R packages "maptools" (Bivand and Lewin-Koh 2016) and "raster" (Hijmans 2012).

\section{Skull form variation}

Morphological data were collected from digital images in the form of landmark configurations covering the skull from its dorsal and ventral side (Figure 2) and described by the set of Cartesian coordinates. The landmark position were digitized in tpsDig 2.18 (Rohlf 2015). The size of each configuration was quantified as a logarithm of its centroid size and its shape was characterized by Procrustes shape coordinates produced by generalized Procrustes analysis (Mitteroecker et al. 2004). The dorsal and ventral skull form (=size and shape) matrices were combined into a single data set and jointly subjected to all analyses.

We tested separately for differences among major phylogeographic lineages and between Aethomys ineptus and the rest of the complex by means of partial least squares (PLS) discrimination analyses with skull form as the predictor and binary coded classification as the response.

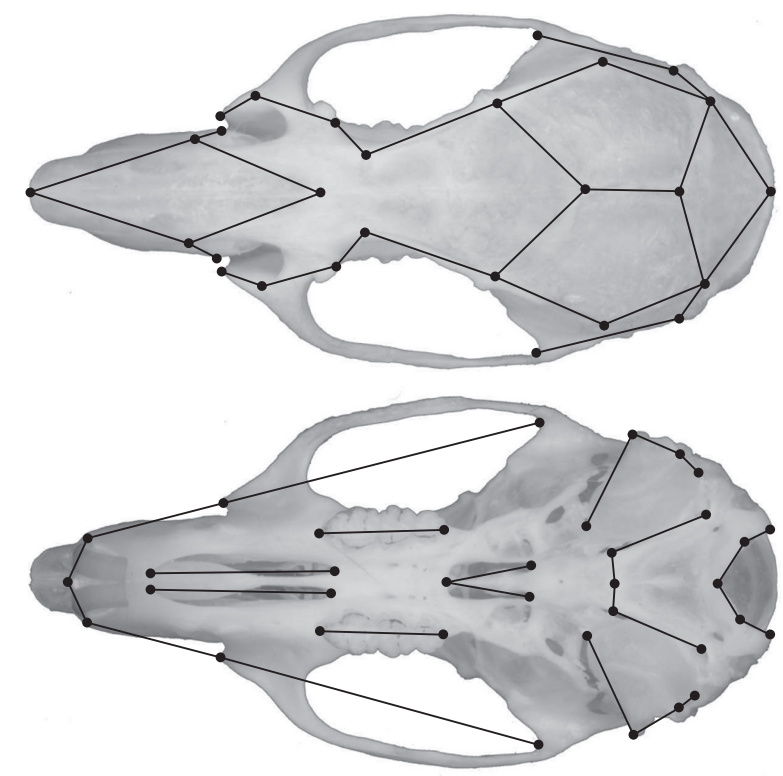

Figure 2: Position of anatomic landmarks on the dorsal and ventral side of the skull.

Landmarks were placed on digital images taken by one of us (OM) in a standardized manner and configurations were then rescaled to units of millimeters. The lines show links between landmarks as displayed when reporting size and shape differences. 
More specifically, we used the canonical powered partial least squares (CPPLS) method of Indahl et al. (2009) as implemented in the R package "pls" (Mevik et al. 2015) with the number of components selected by 10 -fold crossvalidation. The cross-validation was repeated 100 times and we retained the minimum number of components whose median prediction error (PRESS) was within the $50 \%$ highest density interval associated with the minimum median PRESS observed. If at least one component was supported, the differentiation was considered significant and analysed in more detail. In total we analysed 44 adult skulls classified according to their CYTB barcode or, in a few cases, according to their trapping site (see Supplemental File 1). We included material from our collections as well as from four other museums, namely Ditsong Museum of Natural History (Pretoria, South Africa), Royal Museum for Central Africa (Tervuren, Belgium), Naturmuseum Senckenberg (Frankfurt, Germany) and Smithsonian Institution - National Museum of Natural History (Washington, DC, USA).

\section{Results}

\section{Phylogeny, phylogeographic structure, and divergence dating}

Bayesian and maximum likelihood reconstructions of the CYTB gene (Figure 3) showed similar topologies with seven highly supported monophyletic clades labeled A to $\mathrm{G}$ (posterior probability, $\mathrm{PP} \geq 0.98$ and bootstrap support, $\mathrm{BS} \geq 89$ in all cases except for clade $\mathrm{G}$ with $\mathrm{BS}=64$ ). These units were grouped into three larger groups, $\mathrm{A}-\mathrm{D}$ $(\mathrm{PP}=1.00, \mathrm{BS}=99), \mathrm{F}+\mathrm{G}(\mathrm{PP}=0.89, \mathrm{BS}=66)$ and $\mathrm{E}$. Clade E may be a sister clade either to A-D or F+G, where the latter is more probable ( $\mathrm{PP}=0.65)$.

All these clades showed largely parapatric distributional ranges (Figure 1) although the actual distributional limits and possible contact zones are unresolved in some cases due to sampling gaps. Clade A-D occupied the southern part of the distributional range, all of them south of the lower Zambezi - Kafue River system. Clade A corresponded to haplotypes from populations designated as Aethomys ineptus and its presence is evidenced in the north-east of South Africa where it meets clade B south of the Limpopo River. Clade $\mathrm{C}$ was recorded from two sites, one in most western Zimbabwe and the other from the borders of Botswana and Namibia. Clade D was recorded from three sites in southern Zambia, between upper Zambezi and Kafue Rivers. Clade E was

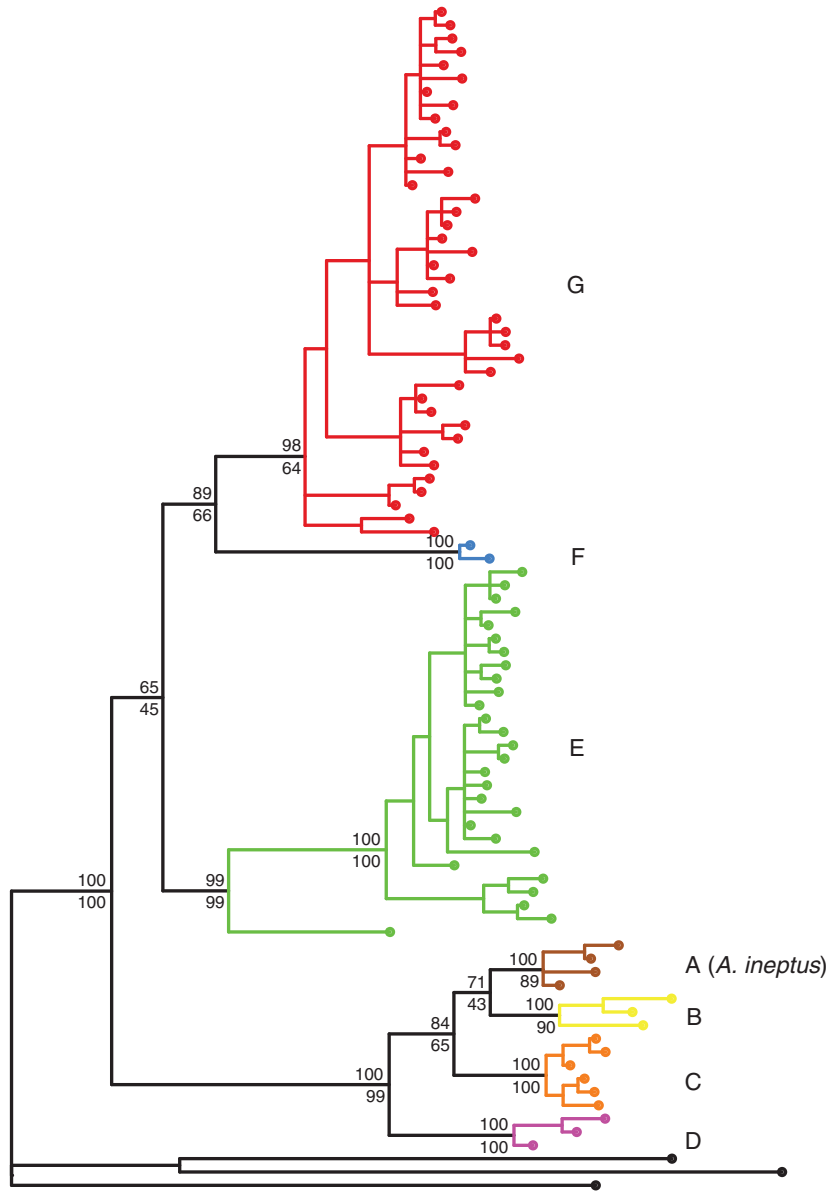

Figure 3: Bayesian tree with nodal values representing: Bayesian posterior probabilities (over the branch) and maximum likelihood bootstrap value (under the branch).

Tip labels are colored as follows: brown for clade A (A. ineptus), yellow for clade $B$, orange for $C$, violet for $D$, green for $E$, blue for $F$, and red for clade $\mathrm{G}$.

documented from central and northern Tanzania and southern-most Kenya, including a distinct haplotype recorded in Namanga Hills $\left(2.53^{\circ} \mathrm{S}, 36.79^{\circ} \mathrm{E}\right)$. Clade G occupied a vast area north of the Zambezi River both west and east of Lake Malawi. This clade was not recorded south of the Zambezi River, but this may be due to sampling gaps in Zimbabwe and central Mozambique where only clade F was recorded from a single site in the Chimanimani mountains. In the northern part of the distribution, clades $\mathrm{G}$ and $\mathrm{E}$ are in contact along the Eastern Arc Mountains and Rukwa Rift in Tanzania. The haplotype network (Figure 4) showed a similar pattern with three very divergent $(E-G)$ and four moderately divergent haplogroups (A-D).

The average phylogenetic distances were $4.01-14.27 \%$ between clades and $0.62-2.95 \%$ within clades and this gap in distribution of pairwise differences made their 


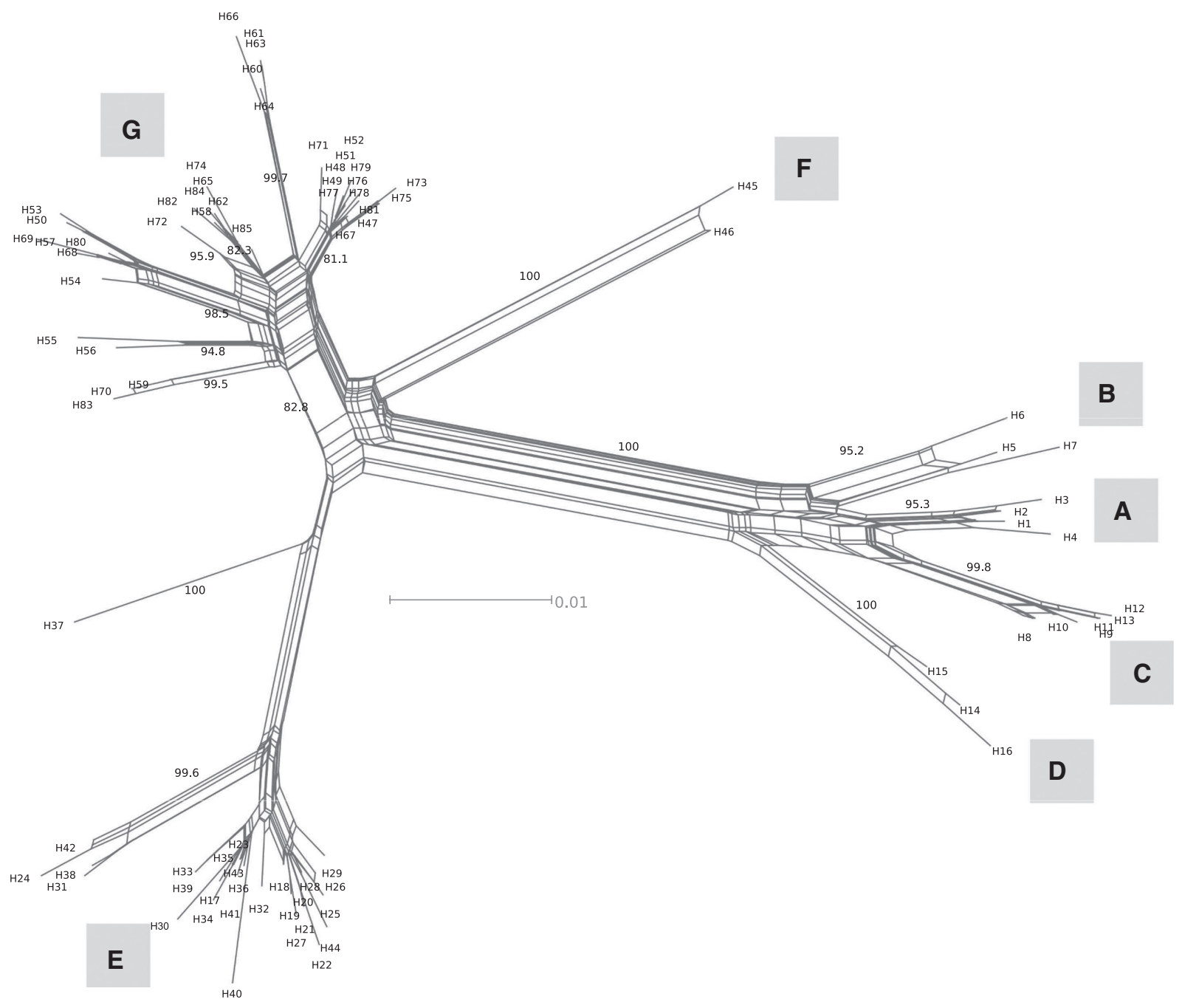

Figure 4: Neighbor-net haplotype network of CYTB sequences of the $A$. chrysophilus complex.

Taxon labels represent haplotype definition and letters corresponding clades. K2P distances were used and the network was drawn using the equal angle method. Bootstrap values (1000 replicates) are shown on selected branches.

delimitation possible. Only clades $\mathrm{E}$ and $\mathrm{G}$ contained more than 10 haplotypes (34 and 70, respectively). Negative values of Tajima's D ( -1.84 and -1.10 , respectively) indicated non-neutral evolution of DNA variation, whereas negative values of Fu's $F_{s}$ (-12.12 and -9.88 , respectively) suggested genetic hitchhiking or population expansion as the likely causes of non-neutrality for both clades. Population expansion was also supported by bell-shaped mismatch distributions (Figure 5) and Harpending raggedness index (0.068 and 0.0067, respectively). All departures of the statistics from zero expectations (and hence null hypothesis of neutrality) were significant at 0.05 level except for Tajima's $\mathrm{D}=-1.10$ in clade $\mathrm{G}(\mathrm{p}=0.12)$.

The root age of the Aethomys phylogeny was estimated at 6.8 Mya with the $95 \%$ highest posterior density (HPD) interval as 6-8.6 Mya (Figure 6). Time to the most recent common ancestor for the Aethomys chrysophilus complex was estimated at $1.3(0.5-2.4)$ Mya and the origin of the Aethomys ineptus lineage at 0.7 (0.1-1.4) Mya. The position of $A$. ineptus inside the $A$. chrysophilus complex was supported with a posterior probability value of 0.94 .

\section{Species distribution modeling}

Our presence records were all included in areas with predicted RORs exceeding uniform prior expectations (Figure 7A) which suggested reasonably good fit of the model. Prediction for the LGM climate produced a distribution map roughly similar to that of current distribution map, albeit more fragmented, especially along the Zambezi River (Figure 7B). On the contrary, prediction for 
A

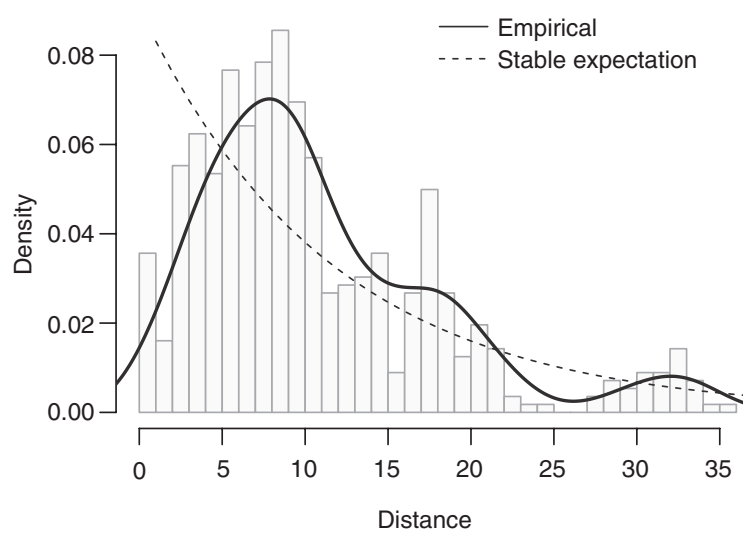

B

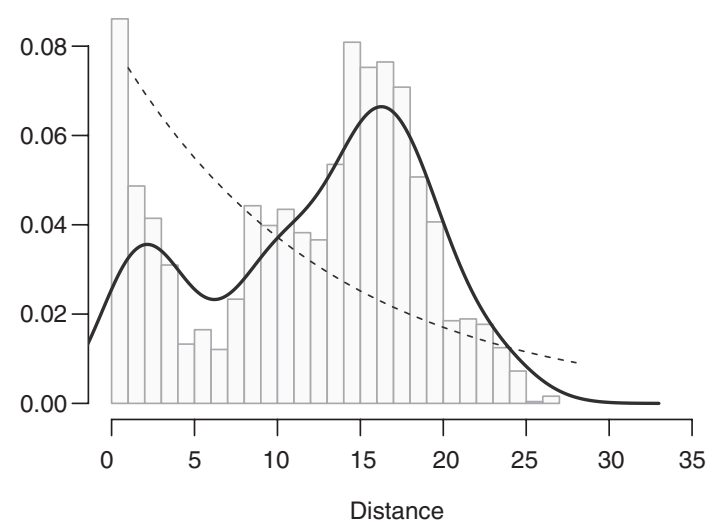

Figure 5: Distribution of the number of pairwise differences among haplotypes in two clades: (A) clade $\mathrm{E}$ and (B) clade G of $A$. chrysophilus. Bars and full line represent the observed distribution and the dotted line represents the expected distribution under the model of stable population fitted to the data.

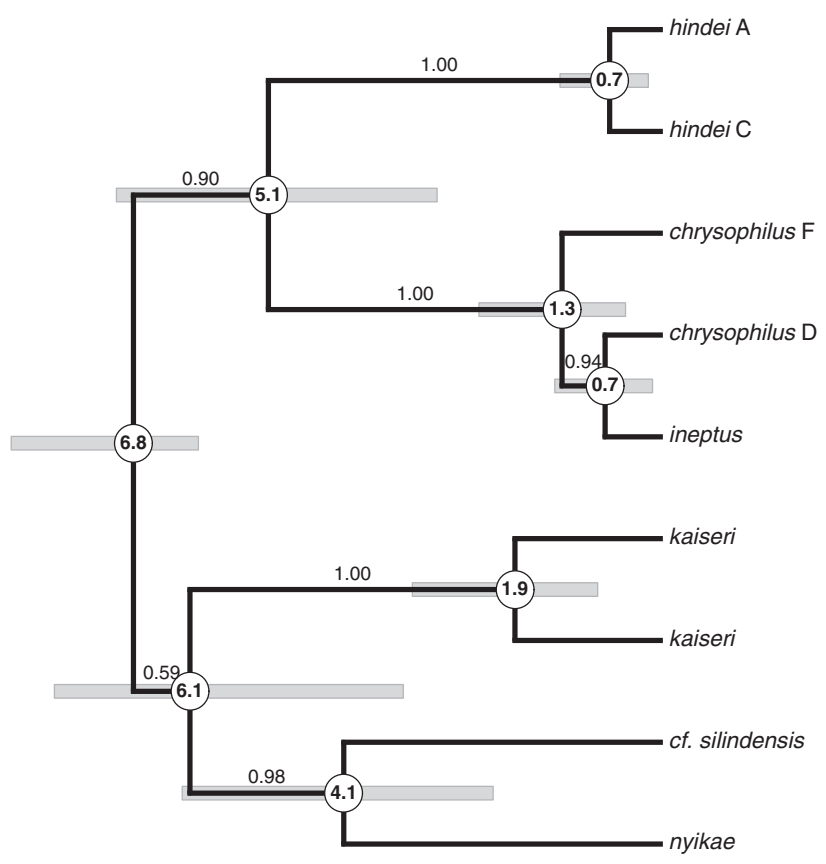

Figure 6: Time calibrated phylogeny of Aethomys based on two nuclear genes, IRBP and RAG1.

Node ages are in Mya, 95\% HPD intervals are shown as light gray rectangles. Posterior probabilities of particular clades are shown above branches supporting them. (Designations "hindei A" and "hindei C" are based on our unpublished phylogeographic data.)

the LIG (Figure 7C) revealed a decrease in suitable conditions across the whole distributional area. In terms of prediction precision loss after randomization the most important variables were: "mean diurnal range of temperatures" (0.68), “mean temperature of driest quarter" $(0.65)$ and "minimum temperature of coldest month" (0.57).
According to comparison of predictor distributions with their ROR-weighted versions (Figure 8) Aethomys chrysophilus avoids areas with high diurnal range of temperatures, especially where combined with high temperature in the cold and dry season. According to WorldClim data (not shown), diurnal range of temperatures is getting too large $\left(>15^{\circ} \mathrm{C}\right)$ in the Kalahari Desert, and the coldest part of the year appears too hot in arid regions of Kenya (min. temperature $>12^{\circ} \mathrm{C}$ ).

\section{Skull form differentiation}

Skull form differentiation among phylogeographic lineages was examined at a coarse scale by comparing only the three main phylogenetic groups: northern (E), central $(F+G)$ and southern (A to D) with sample sizes 15, 19 and 10, respectively. Cross-validation suggested a single component to be retained, which accounted for about $4 \%$ of shape variation, but virtually no size variation in the data set. The cross-validated classification success was low, however, only $67 \%$ for the northern lineage, $63 \%$ for the central lineage and $0 \%$ for the southern one. PLS scores from the final model are shown in Figure 9, suggesting the northern lineage as the most distinct one. Superimposition of shapes predicted for the extreme PLS scores shows the northern lineage as having slightly more robust rostrum and occipital condyles placed more closely to each other (Figure 10). When Aethomys ineptus (six skulls) was contrasted against the rest of the complex, no component was supported as significantly improving on the classification success and skulls form of $A$. ineptus showed no differentiation in our analysis. 

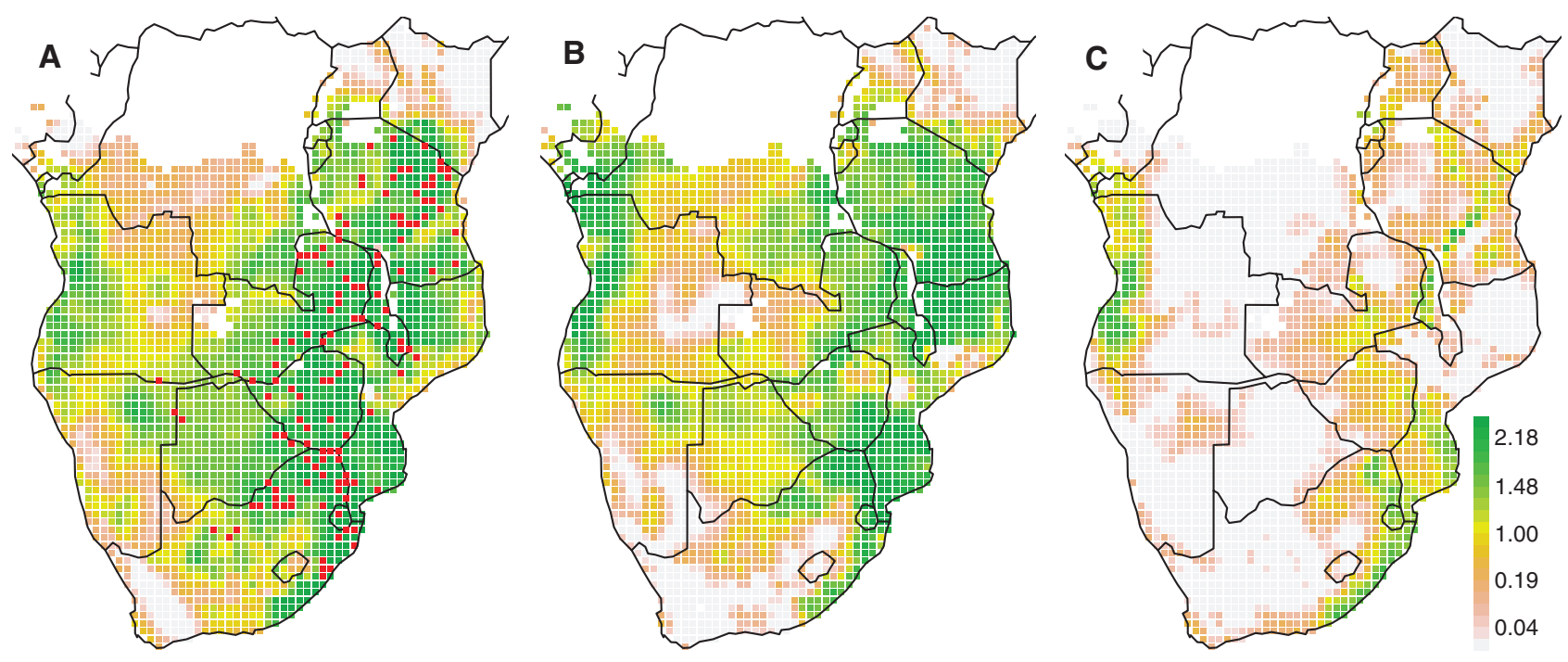

Figure 7: Grid maps of $A$. chrysophilus distribution inferred using maximum entropy model during for (A) current climatic conditions, (B) LGM ( 21,000 years BP) and (C) LIG ( 120,000-140,000 years BP).

Red squares represent sampled coordinates. Dark green color represents preferred conditions, i.e. high RORs relative to prior expectation.

\section{Discussion and conclusion}

\section{Distribution of Aethomys chrysophilus complex}

Our study confirms that Aethomys chrysophilus complex is widely distributed and its habitat requirements are centred mainly to various seasonal savanna-woodland habitats (Skinner and Chimimba 2005, Happold 2013). When suitable habitats are available, these rodents can occur in a wide range of altitudes, from $10 \mathrm{~m}$ a.s.l. (Mozambique, Xai-Xai, $21.12^{\circ} \mathrm{S}, 33.74^{\circ} \mathrm{E}$ ) to about $2300 \mathrm{~m}$ a.s.l. (Tanzania, Mbizi, $7.87^{\circ} \mathrm{S}, 31.67^{\circ} \mathrm{E}$ ). Our distribution modeling supplements this picture with a climate-based quantitative view, which suggests that in spite of its preference for relatively dry habitats, the species avoids extreme conditions reflected by extreme ambient temperatures of deserts or semi-deserts. This result contradicts occurrence of the species in central Kenya, which is indicated by the IUCN Red List but for which we found no evidence in museum collections. Notably, our model predicts extensive occurrence of the $A$. chrysophilus complex along the Atlantic coast in Angola for which we currently have little evidence for in spite of its presence in Namibia and southern Angola (see e.g. Global Biodiversity Information Facility http://www.gbif.org). It would be interesting to examine whether the species occurs in Angola although undocumented in natural history collections and what are its relationships with south-west African endemic Aethomys bocagei (Crawford-Cabral 1998), for which no genetic data are available.

\section{Phylogeographic divides}

In this study we found a pronounced phylogeographic structure within the Aethomys chrysophilus complex where some clades have been separated by well-known biogeographical divides. The distribution of clade $\mathrm{E}$ is delimited by Rukwa Rift and the Eastern Arc Mountains where it meets clade G. These geomorphological features have already been shown to play a prominent role in the history of other rodent taxa living in non-forest habitats such as the pygmy mouse (Mus minutoides, Bryja et al. 2014), the multimammate mouse (Mastomys natalensis, Colangelo et al. 2013, Gryseels et al. 2017), the silvery mole-rat (Heliophobius argenteocinereus, Faulkes et al. 2011) and spiny mice (Acomys spinosissimus complex, Verheyen et al. 2011). In each of these rodent species there are phylogeographic lineages separated either by the Eastern Arc Mountains, the Rukwa Rift or both. The same distributional limits were also observed in an African ungulate species, the bushbuck (Tragelaphus scriptus, Moodley and Bruford 2007).

The contact between clades D and G is located in the area of Kafue Flats, wetland landscape around the Lower Kafue River (Zambia). This area is also transitional for several other species. In bushveld gerbils (Gerbilliscus leucogaster) it forms a barrier to gene flow 
Mean diurnal range of temperatures

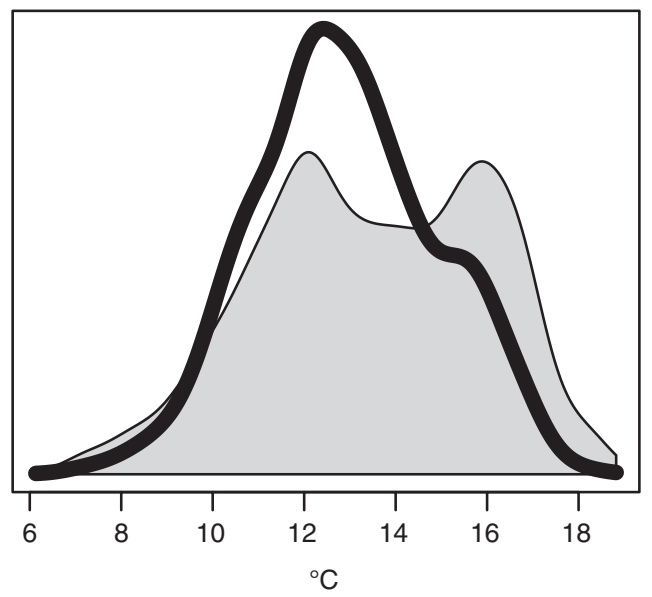

Mean temperature of driest quarter

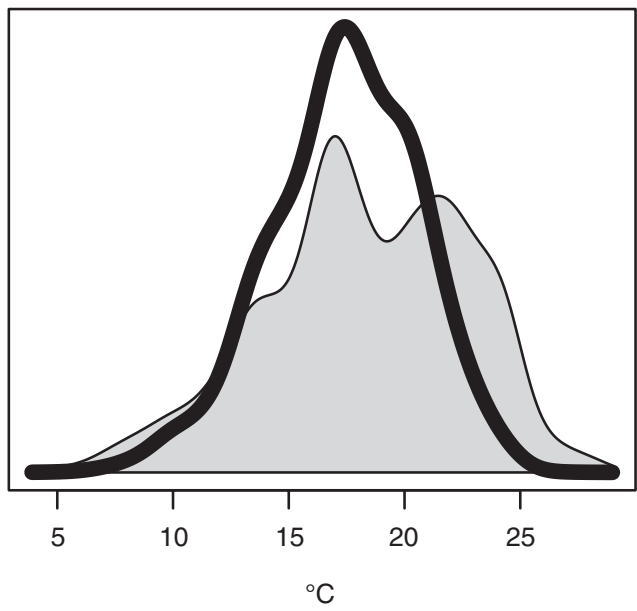

Min temperature of coldest month

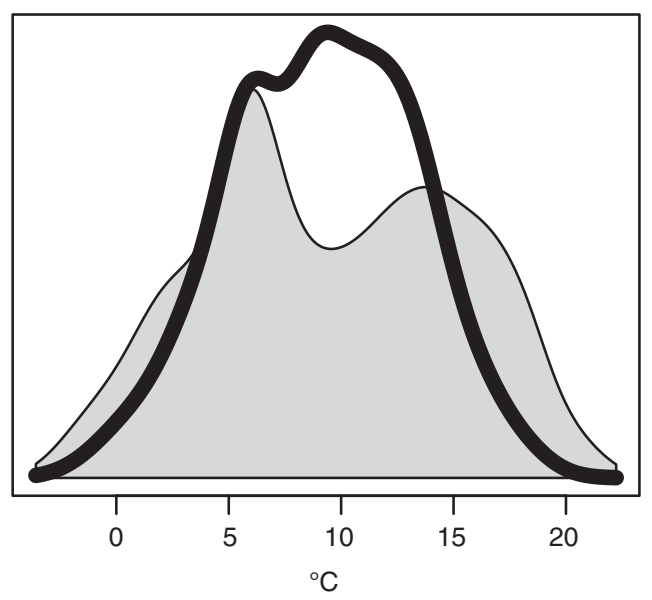

Figure 8: Response plots of three most influential variables in the species distribution model.

Kernel density estimates of background values (in gray) are compared with their ROR-weighted counterparts (in black). In the range of preferred values weighting by the predicted RORs causes an excess of probability density at the expense of less preferred or even avoided intervals.

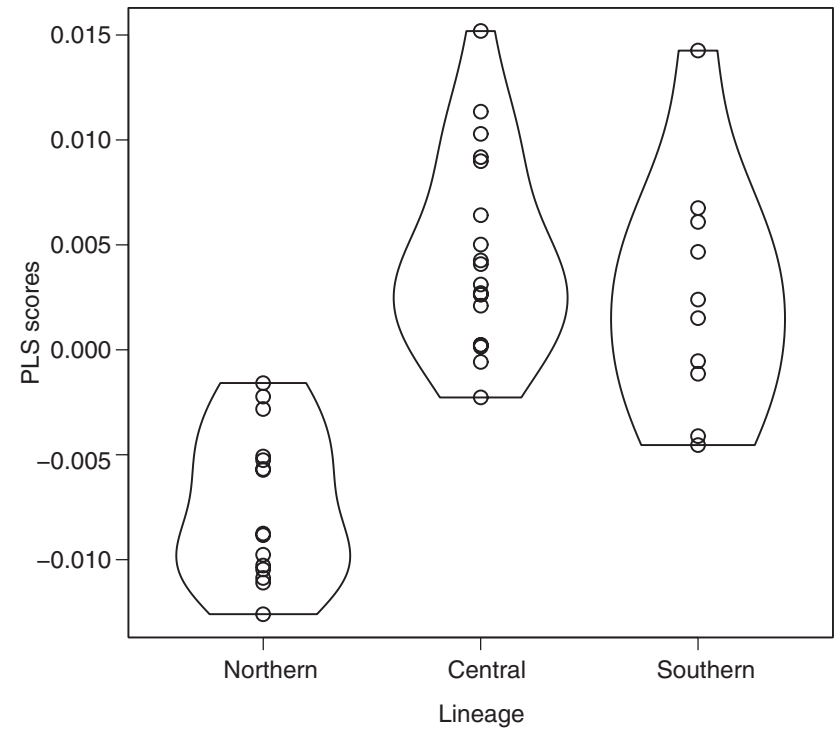

Figure 9: Violin plots showing skull form differentiation among the three major phylogeographic lineages: northern $(E)$, central $(F+G)$ and southern ( $A$ to $D$ ).

Points correspond to individual PLS scores in the final discrimination analysis, envelopes ("violins") are kernel density estimates of their group-specific distributions produced by vioplot package (Adler 2005).
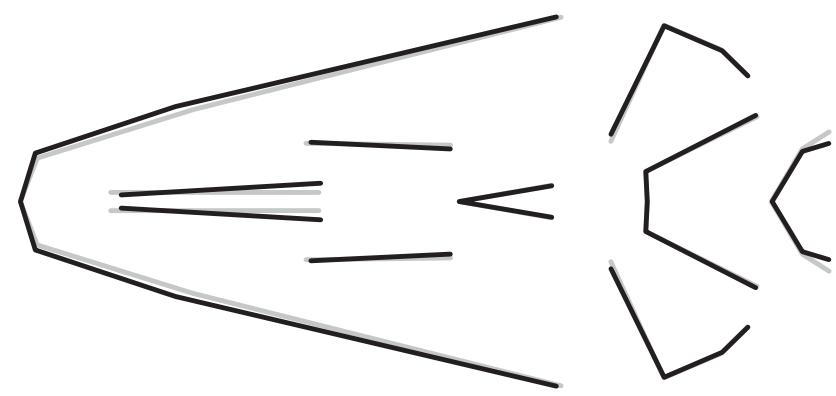

Figure 10: Wireframe plots showing difference in skull shapes (from the ventral view) corresponding to extreme PLS scores.

The shape specific to the northern lineage (in black) is contrasted to the shape specific to the remaining two lineages (in gray).

with observed discordance of nuclear and mitochondrial markers (McDonough et al. 2015) and there is a contact zone between two subspecies of pouched mice (Saccostomus campestris campestris and Saccostomus campestris mashonae, Mikula et al. 2016). From the perspective of large mammals Kafue Flats are part of a wider biogeographical divide in the distribution of baboons (Papio, Zinner et al.2009) and some ungulates including giraffes (Giraffa giraffe, Giraffa tippelskirchi, Fennessy et al. 2016) as well as tsessebe antelopes (Damaliscus) and wildebeests (Connochaetes) as documented by Cotterill (2003). 
The association of parapatric contact zones with mountain ridges or big rivers may be due to partial reproductive isolation, because contact zones with selection against hybrids tend to be associated with migration barriers (Barton and Hewitt 1989). In African rodents, this was suggested by evidence for a hybrid zone between two Saccostomus campestris subspecies at the Zambezi River (Mikula et al. 2016) and between two lineages of Mastomys natalensis along Eastern Arc Mountains (Gryseels et al. 2017). In the Aethomys chrysophilus complex it may be relevant especially in the case of the contact zone between clades A and B in the proximity of the Limpopo River where clade A corresponds to putatively distinct Aethomys ineptus. The actual contact zone seems to have been shifted southward from the river flow (Linzey et al. 2003, Russo et al. 2006) and worth of further investigation. The other two detected contact zones were between lineages $\mathrm{D}-\mathrm{G}$ and $\mathrm{G}-\mathrm{E}$. The contact zone between lineages $\mathrm{G}-\mathrm{E}$ along the Eastern Arc Mountains and Rukwa Rift is well delimited by our present sampling which shows one clade replacing the other over tens of kilometers in spite of the lack of obvious migration barriers.

\section{History of the complex}

Our molecular dating calibrated by the oldest undisputed Aethomys fossil (Lemudong'o, Kenya, 6.1 Mya) and based on two nuclear genes estimated the most recent common ancestor (MRCA) of the genus at 6-8.6 Mya and the MRCA of the Aethomys chrysophilus complex, including the putative speciation of Aethomys ineptus, was estimated at 0.5-2.4 Mya (Pleistocene age). More precisely, the MRCA of the complex is probably $(\mathrm{PP}=0.91)$ younger than 2.0 Mya when tropical climate switched to its modern mode with hydrological cycles controlled by monsoon dynamics (Ravelo et al. 2004). The changes of the African climate and vegetation since that time were driven by interference of high-latitude and low-latitude forcing. Extensive high-latitude glaciations were associated with low sea surface temperatures and low levels of atmospheric $\mathrm{CO}_{2}$. This forced the climate to be cold and dry with grasslands expanding at the expense of forest (deMenocal 1995, Schefuss et al. 2003). This resulted in a complex and regionally asynchronous series of habitat changes (Blome et al. 2012, Shanahan et al. 2015, Johnson et al. 2016) with the potential to cause local extinctions and population divergence.

The observed phylogeographic structure within the Aethomys chrysophilus complex is attributable to the cumulative effect of this extinction-colonization process. First, both well-sampled clades ( $\mathrm{E}$ and $\mathrm{G}$ ) bear signatures of recent population expansion that is either ongoing or young enough to be still apparent in sequence variation. Second, our species distribution model (Figure 7) suggests a range-wide retreat and fragmentation of populations in the LIG $(-120,000-140,000$ years BP). Putative refugia could have been situated north of the Zambezi River in Victoria basin, west of Lake Malawi and along the Eastern Arc Mountains. Pollen records from Lake Malawi indicates open miombo woodland alternating with grassland during drier and wetter phases of the LIG (Beuning et al. 2011) which should support a permanent population of Aethomys chrysophilus in this area. At the same time, forests could separate these putative refugia as they are documented e.g. from the Rukwa rift even during the LGM (Vincens et al. 2005). More extensive inter-glacial distributions are predicted around the Kafue and Luangwa Rivers, south of the Lower Zambezi River and around the Limpopo River including the south.

\section{Skull shape differentiation}

We also found some evidence for skull shape differentiation between the three major phylogeographic lineages. Individuals from the northern lineage (E) inhabiting arid habitats in northwestern Tanzania and southern Kenya had more robust rostrum and differently shaped occipital regions. In terms of cross-validated classification success the differentiation was moderate $(67 \%)$ but significant (PRESS of the null model was outside its 50\% highest density interval). We suggest, therefore, the contrast between lineage $\mathrm{E}$ and the rest as the most likely case of local adaptation within the complex. This should be investigated further by using multiple functional traits.

\section{Taxonomic status of Aethomys ineptus}

Aethomys ineptus is now considered a valid species (Musser and Carleton 2005, Chimimba and Linzey 2008) based on its $2 n=44$ karyotype, aberrant sperm morphology and mitochondrial monophyly. In the rest of the Aethomys chrysophilus complex as well as in other studied species of Aethomys the diploid chromosome number is 50 (Matthey 1958) and spermatozoa resemble those of other murines (Breed 2004). From a systematic and taxonomic point of view A. ineptus may thus represent an interesting case of a species that is monophyletic, defined by clearly derived traits, but phylogenetically embedded in its parental species (a "budding speciation" scenario of Funk and Omland 2003). 
Spermatozoa of Aethomys ineptus differ in many respects including the spatulate-shaped sperm head with no apical hook, modified ultrastructure and shorter tails (Gordon and Watson 1986, Visser and Robinson 1987, Breed et al. 1988). Given the importance of these characteristics in fertilization and postcopulatory selection (Gómez Montoto et al. 2011, Varea-Sánchez et al. 2014) these features may act as a reproductive barrier. The karyotype of $A$. ineptus differs by three centric fusions (Visser and Robinson 1986) that are, by themselves, less likely to cause reproductive incompatibility (Maputla et al. 2011, Dobigny et al. 2015, Medarde et al. 2015), but minor structural changes were also observed. No putative hybrid with intermediate diploid number or any conflicting trait combination has ever been observed (Linzey et al. 2003) which suggests reproductive isolation. On the other hand, distributional patterns of mtDNA and karyotypes are not fully concordant which suggest a degree of genomic admixture. More specifically, both karyotypes were found within the range of our clade A (Visser and Robinson 1986) as well as far beyond it (namely in Zimbabwe, Gordon and Rautenbach 1980). Finally, there may be a mechanistic link between presence of centric fusions and sperm morphology (Medarde et al. 2013) which would make the coincidence of these traits in A. ineptus less surprising but possibly more important.

Morphological traits suggested earlier as diagnostic were not found to be reliable in later studies. Aethomys ineptus has no specific shape of the baculum (Visser and Robinson 1987) and the craniometric delimitation of the species (Chimimba et al. 1999) is in conflict with other evidence (Linzey et al. 2003, this study). Our present analysis also suggests no differentiation in skull size and shape between $A$. ineptus and the rest of the complex. Future studies employing multiple nuclear markers may help to reveal the level of reproductive isolation in the contact zone of both taxa in southern Africa.

Acknowledgments: This study was supported by two projects of the Czech Science Foundation, nos. P506/10/0983 and 15-20229S. We are very grateful to Janice BrittonDavidian and Molly McDonough for providing access to their valuable material. We are indebted to many local authorities for providing necessary permits to carry out our research, especially Zambia Wildlife Authority (ZAWA), National Directorate for Protected Areas (DINAC Mozambique), the Kenyan Forest Service and the Kenyan Wildlife Service, and the Sokoine University of Agriculture in Morogoro, Tanzania. T. Aghová and A. Bryjová helped with genotyping. We express our thanks to our colleagues J. Šklíba, M. Lövy, E. Hrouzková, O. Kott, S. Gryseels,
J. Vrbová Komárková, J. Krásová, G. Phamphi, C. S. Sabuni, A. Katakweba, A. Massawe, as well as protected area officers, rangers and countless local assistants, for the muchneeded support during field expeditions.

\section{References}

Adler, D. 2005. vioplot: Violin plot. R package version 0.2. http:// wsopuppenkiste.wiso.uni-goettingen.de/ dadler.

Aghová, T., R. Šumbera, L. Piálek, O. Mikula, M.M. McDonough, L.A. Lavrenchenko, Y. Meheretu, J.S. Mbau and J. Bryja. 2017. Multilocus phylogeny of east African gerbils (Rodentia, Gerbilliscus) illuminates the history of the Somali-Masai savanna. J. Biogeogr. in press, doi:10.1111/jbi.13017.

Baker, R.J., M.B. Qumsiyeh and I.L. Rautenbach. 1988. Evidence for eight tandem and five centric fusions in the evolution of the karyotype of Aethomys namaquensis A. Smith (Rodentia: Muridae). Genetica 76: 161-169.

Barton, N.H. and G.M. Hewitt. 1989. Adaptation, speciation and hybrid zones. Nature 341: 497-503.

Berger, S.A., D. Krompass and A. Stamatakis. 2011. Performance, accuracy, and web server for evolutionary placement of short sequence reads under maximum likelihood. Syst. Biol. 60: 291-302.

Beuning, K.R.M., K.A. Zimmerman, S.J. Ivory and A.S. Cohen. 2011. Vegetation response to glacial-interglacial climate variability near Lake Malawi in the southern African tropics. Palaeogeogr. Palaeoclimatol. Palaeoecol. 303: 81-92.

Bivand, R. and N. Lewin-Koh. 2016. maptools: tools for Reading and Handling Spatial Objects. https://CRAN.R-project.org/ package $=$ maptools.

Blome, M.W., A.S. Cohen, C.A. Tryon, A.S. Brooks and J. Russell. 2012. The environmental context for the origins of modern human diversity: a synthesis of regional variability in African climate 150,000-30,000 years ago. J. Hum. Evol. 62: 563-592.

Bohoussou, K.H., R. Cornette, B. Akpatou, M. Colyn, J.C. Kerbis Peterhans, J. Kennis, R. Šumbera, E. Verheyen, E. N'Goran, P. Katuala and V. Nicolas. 2015. The phylogeography of the rodent genus Malacomys suggests multiple Afrotropical Pleistocene lowland forest refugia. J. Biogeogr. 42: 2049-2061.

Bouckaert, R.R., J. Heled, D. Kühnert, T. Vaughan, C.H. Wu, D. Xie, M.A. Suchard, A. Rambaut and A.J. Drummond. 2014. BEAST 2: A Software Platform for Bayesian Evolutionary Analysis. PLoS Comput. Biol. 10: e1003537.

Braconnot, P., B. Otto-Bliesner, S. Harrison, S. Joussaume, J.-Y. Peterchmitt, A. Abe-Ouchi, M. Crucifix, E. Driesschaert, T. Fichefet, C.D. Hewitt, M. Kageyama, A. Kitoh, A. Laîné, M.-F. Loutre, O. Marti, U. Merkel, G. Ramstein, P. Valdes, S.L. Weber, Y. Yu and Y. Zhao. 2007. Results of PMIP2 coupled simulations of the Mid-Holocene and Last Glacial Maximum - Part 1: experiments and large-scale features. Clim. Past. 3: 261-277.

Breed, W.G. 2004. The spermatozoon of eurasian murine rodents: Its morphological diversity and evolution. J. Morphol. 261: 52-69.

Breed, W.G., G.A. Cox, C.M. Leigh and P. Hawkins. 1988. Sperm head structure of a murid rodent from southern Africa: the red veld rat Aethomys chrysophilus. Gamete Res. 19: 191-202. 
Bryja, J., L. Granjon, G. Dobigny, H. Patzenhauerová, A. Konečný, J.M. Duplantier, P. Gauthier, M. Colyn, L. Durnez, A. Lalis and V. Nicolas. 2010. Plio-Pleistocene history of West African Sudanian savanna and the phylogeography of the Praomys daltoni complex (Rodentia): the environment/geography/genetic interplay. Mol. Ecol. 19: 4783-4799.

Bryja, J., O. Mikula, R. Šumbera, Y. Meheretu, T. Aghová, L.A. Lavrenchenko, V. Mazoch, N. Oguge, J.S. Mbau, K. Welegerima, N. Amundala, M. Colyn, H. Leirs and E. Verheyen. 2014. PanAfrican phylogeny of Mus (subgenus Nannomys) reveals one of the most successful mammal radiations in Africa. BMC Evol. Biol. 14: 256.

Castiglia, R., M. Corti, P. Colangelo, F. Annesi, E. Capanna, W. Verheyen, A.M. Sichilima and R. Makundi. 2003. Chromosomal and molecular characterization of Aethomys kaiseri from Zambia and Aethomys chrysophilus from Tanzania (Rodentia, Muridae). Hereditas. 139: 81-89.

Chimimba, C.T. 1998. A taxonomic synthesis of southern African Aethomys (Rodentia: Muridae) with a key to species. Mammalia 62: 427-438.

Chimimba, C.T. and A. V. Linzey. 2008. Aethomys ineptus (Rodentia: Muridae). Mamm. Species. 809: 1-7.

Chimimba, C.T., N.J. Dippenaar and T.J. Robinson. 1999.

Morphometric and morphological delineation of southern African species of Aethomys (Rodentia: Muridae). Biol. J. Linn. Soc. 67: 501-527.

Colangelo, P., E. Verheyen, H. Leirs, C. Tatard, C. Denys, G. Dobigny, J.M. Duplantier, C. Brouat, L. Granjon and E. Lecompte. 2013. A mitochondrial phylogeographic scenario for the most widespread African rodent, Mastomys natalensis. Biol. J. Linn. Soc. 108: 901-916.

Cotterill, F.P.D. 2003. A biogeographic review of tsessebe antelopes, Damaliscus lunatus (Bovidae: Alcelaphini), in south-central Africa. Durban Museum Novit. 28: 45-55.

Crawford-Cabral, J. 1998. The Angolan rodents of the superfamily Muroidea: an account of their distribution. Instituto de Investigação Científica Tropical, Estudos, Ensaios e Documentos, Vol. 161, pp. 1-222.

Darriba, D., G.L. Taboada, R. Doallo and D. Posada. 2012. jModelTest 2: more models, new heuristics and parallel computing. Nat. Methods. 9: 772 .

Deino, A.L. and S.H. Ambrose. 2007. 40Ar/39Ar dating of the Lemudong'o late Miocene fossil assemblages, southern Kenya rift. Kirtlandia. 56: 65-71.

deMenocal, P.B. 1995. Plio-Pleistocene African climate. Science 270: 53-59.

Demos, T.C., J.C. Kerbis Peterhans, B. Agwanda and M.J. Hickerson. 2014. Uncovering cryptic diversity and refugial persistence among small mammal lineages across the Eastern Afromontane biodiversity hotspot. Mol. Phylogenet. Evol. 71: 41-54.

Dobigny, G., C. Tatard, P. Gauthier, K. Ba, J.-M. Duplantier, L. Granjon and G.J. Kergoat. 2013. Mitochondrial and nuclear genes-based phylogeography of Arvicanthis niloticus (Murinae) and sub-Saharan open habitats pleistocene history. PLoS One 8: e77815.

Dobigny, G., J. Britton-Davidian and T.J. Robinson. 2015. Chromosomal polymorphism in mammals: an evolutionary perspective. Biol. Rev. 92: 1-21.

Drummond, A.J., S.Y.W. Ho, M.J. Phillips and A. Rambaut. 2006. Relaxed Phylogenetics and Dating with Confidence. PLoS Biol. 4: e88.
Ducroz, J.F., V. Volobouev and L. Granjon. 2001. An assessment of the systematics of Arvicanthine rodents using mitochondrial DNA sequences: evolutionary and biogeographical implications. J. Mamm. Evol. 8: 173-206.

Fadda, C., R. Castiglia, P. Colangelo, M. Corti, R. Machang'u, A. Scanzani, P. Tesha, W. Verheyen and E. Capanna. 2001. The rodent fauna of Tanzania: a cytotaxonomic report from the Maasai Steppe (1999). Rend. Fis. Acc. Lincei 9: 29-49.

Faulkes, C.G., N.C. Bennett, F.P.D. Cotterill, W. Stanley, G.F. Mgode and E. Verheyen. 2011. Phylogeography and cryptic diversity of the solitary-dwelling silvery mole-rat, genus Heliophobius (family: Bathyergidae). J. Zool. 285: 324-338.

Fennessy, J., T. Bidon, F. Reuss, V. Kumar, P. Elkan, M.A. Nilsson, M. Vamberger, U. Fritz and A. Janke. 2016. Multi-locus analyses reveal four giraffe species instead of one. Curr. Biol. 26: 1-7.

Fu, Y.X. 1997. Statistical tests of neutrality of mutations against population growth, hitchhiking and background selection. Genetics. 147: 915-925.

Funk, D.J. and K.E. Omland. 2003. Species-level paraphyly and polyphyly: frequency, causes, and consequences, with insights from animal mitochondrial DNA. Annu. Rev. Ecol. Evol. Syst. 34: 397-423.

Galan, M., M. Pagès and J.F. Cosson. 2012. Next-generation sequencing for rodent barcoding: species identification from fresh, degraded and environmental samples. PLoS One 7: e48374.

Galster, S., N.D. Burgess, J. Fjeldså, L.A. Hansen and C. Rahbek. 2007. One degree resolution databases of the distribution of 1085 species of mammals in Sub-Saharan Africa. On-line data source-Version 1.00. Zoological Museum, University of Copenhagen, Denmark. Available via http://www.daim.snm.ku.dk/ african-vertebrates-searc.

Geraads, D., Z. Alemseged and H. Bellon. 2002. The late Miocene mammalian fauna of Chorora, Awash basin, Ethiopia: systematics, biochronology and 40K-40Ar ages of the associated volcanics. Tert. Res. 21: 113-122.

Gómez Montoto, L., C. Magaña, M. Tourmente, J. Martín-Coello, C. Crespo, J.J. Luque-Larena, M. Gomendio and E.R.S. Roldan. 2011. Sperm competition, sperm numbers and sperm quality in muroid rodents. PLoS One 6: e18173.

Gordon, D.H. and I.L. Rautenbach. 1980. Species complexes in medically important rodents: chromosome studies of Aethomys, Tatera and Saccostomus (Rodentia: Muridae, Cricetidae). S. Afr. J. Sci. 76: 559-561.

Gordon, D.H. and C.R.B. Watson. 1986. Identification of cryptic species of rodents (Mastomys, Aethomys, Saccostomus) in the Kruger National Park. South African J. Zool. 21: 95-99.

Gryseels, S., S.J.E. Baird, B. Borremans, R. Makundi, H. Leirs and J. Goüy de Bellocq. 2017. When viruses don't go viral: the importance of host phylogeographic structure in the spatial spread of arenaviruses. PLoS Pathogens 13: e1006073.

Happold, D.C.D. (ed.). 2013. Volume III. Rodents, Hares and Rabbits. Bloomsbury Publishing, London. p. 789.

Haus, T., E. Akom, B. Agwanda, M. Hofreiter, C. Roos and D. Zinner. 2013. Mitochondrial diversity and distribution of African Green Monkeys (Chlorocebus Gray, 1870). Am. J. Primatol. 75: 350-360.

Heled, J. and A.J. Drummond. 2012. Calibrated tree priors for relaxed phylogenetics and divergence time estimation. Syst. Biol. 61: 138-149. 
Hijmans, R.J. 2012. Introduction to the 'raster' package (version 1.9-63). https://CRAN.R-project.org/package=raster.

Hijmans, R.J., S.E. Cameron, J.L. Parra, P.G. Jones and A. Jarvis. 2005. Very high resolution interpolated climate surfaces for global land areas. Int. J. Climatol. 25: 1965-1978.

Hijmans, R.J., S. Phillips, J. Leathwick and J. Elith. 2016. Dismo: species distribution modeling. https://CRAN.R-project.org/ package $=$ dismo.

Huson, D.H. and D. Bryant. 2006. Application of phylogenetic networks in evolutionary studies. Mol. Biol. Evol. 23: 254-267.

Indahl, U.G., K.H. Liland and T. Næs. 2009. Canonical partial least squares-a unified PLS approach to classification and regression problems. J. Chemom. 23: 495-504.

Irwin, D.M., T.D. Kocher and A.C. Wilson. 1991. Evolution of cytochrome-b gene in mammals. J. Mol. Evol. 32: 128-144.

Johnson, T.C., J.P. Werne, E.T. Brown, A. Abbott, M. Berke, J. Halbur, S. Contreras - Quintana, S. Grossheusch, S. Schouten, J. Sinninghe Damsté, R. Lyons, C.A. Scholz, A. Cohen and J. King. 2016. A progressively wetter climate in Southern East Africa over the past million years. Quat. Int. 404: 174-175.

Kimura, M. 1980. A simple method for estimating evolutionary rates of base substitutions through comparative studies of nucleotide sequences. J. Mol. Evol. 16: 111-120.

Lehner, B. and P. Döll. 2004. Development and validation of a global database of lakes, reservoirs and wetlands. J. Hydrol. 296: 1-22.

Librado, P. and J. Rozas. 2009. DnaSP v5: a software for comprehensive analysis of DNA polymorphism data. Bioinformatics. 25: 1451-1452.

Linzey, A.V. and C.T. Chimimba. 2008. Aethomys chrysophilus (Rodentia: Muridae). Mamm. Species. 808: 1-10.

Linzey, A.V., M.H. Kesner, C.T. Chimimba and C. Newbery. 2003. Distribution of veld rat sibling species Aethomys chrysophilus and Aethomys ineptus (Rodentia: Muridae) in southern Africa. African Zool. 38: 169-174.

Lorenzen, E.D., R. Heller and H.R. Siegismund. 2012. Comparative phylogeography of African savannah ungulates. Mol. Ecol. 21: 3656-3670.

Manthi, F.K. 2007. A preliminary review of the rodent fauna from Lemudong'o, southwestern Kenya, and its implication to the late Miocene paleoenvironments. Kirtlandia. 56: 92-105.

Maputla, N.W., E.R. Dempster, J. Raman and J.W.H. Ferguson. 2011. Strong hybrid viability between two widely divergent chromosomal forms of the pouched mouse. J. Zool. 285: 180-187.

Matthey, R. 1958. Les chromosomes et la position systématique de quelques Murinae africains (Mammalia Rodentia). Acta Trop. 15: 7-117.

McDonough, M.M., R. Šumbera, V. Mazoch, A.W. Ferguson, C.D. Phillips and J. Bryja. 2015. Multilocus phylogeography of a widespread savanna-woodland adapted rodent reveals the influence of Pleistocene geomorphology and climate change in Africa's Zambezi region. Mol. Ecol. 24: 5248-5266.

Medarde, N., J. Martínez-Vargas, A. Sánchez-Chardi, M.J. LópezFuster and J. Ventura. 2013. Effect of Robertsonian translocations on sperm head form in the house mouse. Biol. J. Linn. Soc. 110: 878-889.

Medarde, N., V. Merico, M.J. López-Fuster, M. Zuccotti, S. Garagna and J. Ventura. 2015. Impact of the number of Robertsonian chromosomes on germ cell death in wild male house mice. Chromosom. Res. 23: 159-169.
Mein, P., M. Pickford and B. Senut. 2004. Late Miocene micromammals from the Harasib karst deposits, Namibia. Pt. 2b. Cricetomyidae, Dendromuridae and Muridae, with an addendum on the Myocricetodontinae. Commun. Geol. Surv. Namibia. 13: 43-62.

Merow, C., M.J. Smith and J.A. Silander. 2013. A practical guide to MaxEnt for modeling species' distributions: what it does, and why inputs and settings matter. Ecography (Cop.). 36: 1058-1069.

Mevik, B.-H., R. Wehrens and K.H. Liland. 2015. pls: Partial Least Squares and Principal Component Regression. https://CRAN.Rproject.org $/$ package=pls.

Mikula, O., R. Šumbera, T. Aghová, J.S. Mbau, A.S. Katakweba, C.A. Sabuni and J. Bryja. 2016. Evolutionary history and species diversity of African pouched mice (Rodentia: Nesomyidae: Saccostomus). Zool. Scr. 45: 595-617.

Miller, M.A., W. Pfeiffer and T. Schwartz. 2010. Creating the CIPRES Science Gateway for inference of large phylogenetic trees. In: (Gateway Computing Environments Workshop (GCE), 2010). IEEE, New Orleans, LA, USA, pp. 1-8.

Mitteroecker, P., P. Gunz, M. Bernhard, K. Schaefer and F.L. Bookstein. 2004. Comparison of cranial ontogenetic trajectories among great apes and humans. J. Hum. Evol. 46: 679-698.

Moodley, Y. and M.W. Bruford. 2007. Molecular biogeography: towards an integrated framework for conserving Pan-African biodiversity. PLoS One 2: e454.

Muscarella, R., P.J. Galante, M. Soley-Guardia, R.A. Boria, J.M. Kass, M. Uriarte and R.P. Anderson. 2014. ENMeval: an R package for conducting spatially independent evaluations and estimating optimal model complexity for MAXENT ecological niche models. Methods Ecol. Evol. 5: 1198-1205.

Musser, G.G. and M.D. Carleton. 2005. Superfamily Muroidea. In: (D.E., Wilson and D.M. Reeder, eds.) Mammal species of the world: a taxonomic and geographic reference. 3rd ed. Johns Hopkins University Press, Baltimore, MD. pp. 894-1531.

Nicolas, V., J.F. Mboumba, E. Verheyen, C. Denys, E. Lecompte, A. Olayemi, A.D. Missoup, P. Katuala and M. Colyn. 2008. Phylogeographic structure and regional history of Lemniscomys striatus (Rodentia: Muridae) in tropical Africa. J. Biogeogr. 35: 2074-2089.

Nicolas, V., A.D. Missoup, C. Denys, J.C. Kerbis Peterhans, P. Katuala, A. Couloux and M. Colyn. 2011. The roles of rivers and Pleistocene refugia in shaping genetic diversity in Praomys misonnei in tropical Africa. J. Biogeogr. 38: 191-207.

Olson, D.M., E. Dinerstein, E.D. Wikramanayake, N.D. Burgess, G.V.N. Powell, E.C. Underwood, J.A. D’amico, I. Itoua, H.E. Strand, J.C. Morrison, C.J. Loucks, T.F. Allnutt, T.H. Ricketts, Y. Kura, J.F. Lamoreux, W.W. Wettengel, P. Hedao, K.R. Kassem, E.D. Wikramanaya, N.D. Burgess, G.V.N. Powell, E.C. Underwood, J.A. D’amico, I. Itoua, H.E. Strand, J.C. Morrison, C.J. Loucks, T.F. Allnutt, T.H. Ricketts, Y. Kura, J.F. Lamoreux, W.W. Wettengel, P. Hedao and K.R. Kassem. 2001. Terrestrial ecoregions of the world: a new map of life on earth. Bioscience. 51: 933.

Otto-Bliesner, B.L., S.J. Marshall, J.T. Overpeck, G.H. Miller and A. Hu. 2006. Simulating Arctic climate warmth and icefield retreat in the last interglaciation. Science 311: 1751-1753.

Paradis, E. 2010. Pegas: an R package for population genetics with an integrated-modular approach. Bioinformatics 26: 419-420. 
Paradis, E., J. Claude and K. Strimmer. 2004. APE: analyses of phylogenetics and evolution in $\{R\}$ language. Bioinformatics 20: 289-290.

Phillips, S.J., R.P. Anderson and R.E. Schapire. 2006. Maximum entropy modeling of species geographic distributions. Ecol. Modell. 190: 231-259.

Phukuntsi, M.A., H. Brettschneider, D.L. Dalton, T. Kearney, J. Badenhorst and A. Kotze. 2016. DNA barcoding for identification of cryptic species in the field and existing museum collections: a case study of Aethomys and Micaelamys (Rodentia: Muridae). African Zoology 2016: 1-8.

Rambaut, A. and A.J. Drummond. 2013. Tracer 1.6. University of Edinburgh, Edinburgh.

Ravelo, A.C., D.H. Andreasen, M. Lyle, A. Olivarez Lyle and M.W. Wara. 2004. Regional climate shifts caused by gradual global cooling in the Pliocene epoch. Nature 429: 263-267.

R Core Team. 2016. R: a language and environment for statistical computing.

Rogers, A.R. and H. Harpending. 1992. Population growth makes waves in the distribution of pairwise genetic differences. Mol. Biol. Evol. 9: 552-569.

Rohlf, F.J. 2015. tpsDig 2.18 Department of Ecology and Evolution, State University of New York, Stony Brook. Avalibale at: http:// life.bio.sunysb.edu/morph.

Ronquist, F., M. Teslenko, P. van der Mark, D.L. Ayres, A. Darling, S. Höhna, B. Larget, L. Liu, M.A. Suchard and J.P. Huelsenbeck. 2012. MrBayes 3.2: efficient Bayesian phylogenetic inference and model choice across a large model space. Syst. Biol. 61: 539-542.

Russo, I.M., C.T. Chimimba and P. Bloomer. 2006. Mitochondrial DNA differentiation between two species of Aethomys (Rodentia: Muridae) from southern Africa. J. Mammal. 87: 545-553.

Russo, I.M., C.T. Chimimba and P. Bloomer. 2010. Bioregion heterogeneity correlates with extensive mitochondrial DNA diversity in the Namaqua rock mouse, Micaelamys namaquensis (Rodentia: Muridae) from southern Africa - evidence for a species complex. BMC Evol. Biol. 10: 307.

Schefuss, E., S. Schouten, J.H.F. Jansen and J.S.S. Damsté. 2003. African vegetation controlled by tropical sea surface temperatures in the mid-Pleistocene period. Nature 422: 418-421.

Shanahan, T.M., N.P. McKay, K.A. Hughen, J.T. Overpeck, B. OttoBliesner, C.W. Heil, J. King, C.A. Scholz and J. Peck. 2015. The time-transgressive termination of the African Humid Period. Nat. Geosci. 8: 140-144.

Skinner, J.D. and C.T. Chimimba. 2005. The mammals of the southern African sub-region (3rd ed.). Cambridge University Press, Cambridge. p. 872.
Stamatakis, A. 2014. RAxML version 8: A tool for phylogenetic analysis and post-analysis of large phylogenies. Bioinformatics 30: 1312-1313.

Stamatakis, A., P. Hoover and J. Rougemont. 2008. A rapid bootstrap algorithm for the RAxML Web servers. Syst. Biol. 57: 758-771.

Stanhope, M.J., J. Czelusniak, J.-S. Si, J. Nickerson and M. Goodman. 1992. A molecular perspective on mammalian evolution from the gene encoding interphotoreceptor retinoid binding protein, with convincing evidence for bat monophyly. Mol. Phylogenet. Evol. 1: 148-160.

Suwa, G., Y. Beyene, H. Nakaya, R.L. Bernor, J.-R. Boisserie, F. Bibi, S.H. Ambrose, K. Sano, S. Katoh and B. Asfaw. 2015. Newly discovered cercopithecid, equid and other mammalian fossils from the Chorora Formation, Ethiopia. Anthropol. Sci. 123: 19-39.

Tajima, F. 1989. Statistical method for testing the neutral mutation hypothesis by DNA polymorphism. Genetics 123: 585-595.

Teeling, E.C., M. Scally, D.J. Kao, M.L. Romagnoli, M.S. Springer, and M.J. Stanhope. 2000. Molecular evidence regarding the origin of echolocation and flight in bats. Nature 403: 188-192.

Varea-Sánchez, M., L. Gómez Montoto, M. Tourmente and E.R.S. Roldan. 2014. Postcopulatory sexual selection results in spermatozoa with more uniform head and flagellum sizes in rodents. PLoS One 9: e108148.

Verheyen, W., J. Hulselmans, W. Wendelen, H. Leirs and M. Corti. 2011. Contribution to the systematics and zoogeography of the East-African Acomys. Zootaxa. 3059: 1-35.

Vincens, A., G. Buchet, D. Williamson and M. Taieb. 2005. A 23,000 yr pollen record from Lake Rukwa ( $8^{\circ} \mathrm{S}$, SW Tanzania): New data on vegetation dynamics and climate in Central Eastern Africa. Rev. Palaeobot. Palynol. 137: 147-162.

Visser, D.S. and T.J. Robinson. 1986. Cytosystematics of the South African Aethomys (Rodentia: Muridae). South African J. Zool. 21: 264-268.

Visser, D.S. and T.J. Robinson. 1987. Systematic implications of spermatozoan and bacular morphology for the South African Aethomys. Mammalia 51: 447-454.

Warren, D.L. and S.N. Seifert. 2011. Ecological niche modeling in Maxent: the importance of model complexity and the performance of model selection criteria. Ecol. Appl. 21: 335-342.

Zinner, D., L.F. Groeneveld, C. Keller and C. Roos. 2009. Mitochondrial phylogeography of baboons (Papio spp.): indication for introgressive hybridization? BMC Evol. Biol. 9: 83.

Zinner, D., J. Wertheimer, R. Liedigk, L.F. Groeneveld and C. Roos. 2013. Baboon phylogeny as inferred from complete mitochondrial genomes. Am. J. Phys. Anthropol. 150: 133-140.

Supplemental Material: The online version of this article offers supplementary material (https://doi.org/10.6084/m9.figshare. 4516745 and https://doi.org/10.6084/m9.figshare.4960406). 